ALZIRA XAVIER GARcês ${ }^{1}$

ana Maria Barral de Martinez ${ }^{3}$

Carla Vitola Gonçalves ${ }^{3}$

Fabiana Nunes Germano ${ }^{2}$

Maria Fernanda Martinez BarRal ${ }^{1}$

VAlDimara CorReia Vieira ${ }^{2}$

Artigo Original

Palavras-chave

Infecções por Chlamydia Doenças sexualmente transmissíveis Reação em cadeia da polimerase

Fatores de risco

Questionários

Prevalência

Keywords

Chlamydia infections Sexually transmitted diseases Polymerase chain reaction Risk factors

Questionnaires Prevalence

\title{
Prevalência de Chlamydia trachomatis e fatores de risco associados à infecção detectada em amostra endocervical
}

\author{
Prevalence of Chlamydia trachomatis and risk factors \\ associated with infection detected in endocervical sample
}

\section{Resumo}

OBJETIVO: Foi avaliar a prevalência de Chlamydia trachomatis e os fatores de risco associados à infecção em amostras endocervicais de mulheres atendidas em ambulatório de Ginecologia e Obstetrícia. MÉTODOS: Amostras de secreção endocervical de 200 mulheres atendidas em Hospital Universitário foram avaliadas para diagnosticar C. trachomatis com uso da reação em cadeia da polimerase (PCR) utilizando primers CT05/CTO6 que amplificam 281 pares de bases da principal proteína de membrana externa de C. trachomatis. Todas as participantes responderam a um questionário pré-codificado e autoaplicável. Os dados foram analisados no programa do software SPSS 17.0; para a análise multivariada foi utilizada a regressão de Poisson. RESULTADOS: Das 200 mulheres que foram incluídas no estudo, a prevalência de infecção por C. trachomatis foi de 1 1\% (22 pacientes) e destas 55 (27,5\%) foram positivas para o HPV. Os fatores de risco associados à infecção por C. trachomatis foram: ter 8 anos ou menos de escolaridade $(p<0,001)$, renda familiar de até 1 salário mínimo ( $p=0,005)$, primeira relação sexual com 15 anos ou menos $(p=0,04)$ e ser portadora do vírus HIV $(p<0,001)$. Após a análise multivariada, apenas as variáveis escolaridade igual ou inferior a oito anos (RP 6,0; IC95\% 1,26-29,0; $\mathrm{p}=0,02$ ) e presença do HIV (RP 14, 1; IC95\% 3,4-57,5; $p<0,0011$ permaneceram significantes. CONCLUSÕES: A prevalência de C. trachomatis em amostras endocervicais pelo método de PCR foi de $11 \%$. Os fatores associados à maior infecção por C. trachomatis foram menor escolaridade e ser portar o vírus HIV.

\section{Abstract}

PURPOSE: It was to determine the prevalence of Chlamydia trachomatis and the risk factors associated with infection in endocervical specimens from women seen in outpatient Obstetrics and Gynecology. METHODS: Samples of endocervical secretion of 200 women treated at the University Hospital of the Federal University of Rio Grande were analyzed for the presence of $C$. trachomatis by polymerase chain reaction (PCR) using primers that amplify CT05/ CTO6 281 base pairs of the main outer membrane protein of $C$. trachomatis. All participants completed a pre-coded and self-report questionnaire. Data were analyzed with the SPSS 17.0 software; for multivariate analysis it was used Poisson regression. RESULTS: Of the 200 women who were included in the study, the prevalence of infection with $C$. trachomatis was 1 1\% (22 patients) and these 55 (27.5\%) were positive for HPV. Risk factors associated with infection by $C$. trachomatis were: 8 years or less of schooling ( $p<0.001$ ), family income below the poverty level ( $p=0.005)$, first intercourse at age 15 or less $(\mathrm{p}=0.04)$ and being a carrier of the virus $\mathrm{HIV}(\mathrm{p}<0.001)$. After multivariate analysis, only the variables of schooling or less than eight years (PR 6.0; 95\% $1.26-29.0 ; \mathrm{p}=0.02$ ) and presence of HIV (RP 14.1; $95 \% \mathrm{Cl}$ 3.4-57.5; $\mathrm{p}<0.001$ ) remained statistically significant. CONCLUSIONS: The prevalence of $\mathrm{C}$. trachomatis in endocervical specimens by PCR was $11 \%$. The factors associated with a higher infection by $C$. trachomatis were lower education and being HIV positive.

Correspondência

Carla Vitola Gonçalves Faculdade de Medicina da Universidade Federal do Rio Grande Rua Generol 0sório, $s / n-4^{\circ}$ anda CEP: $96201-900$ Rio Grande (RS), Brasil

Recebido $16 / 07 / 2013$

Aceito com modificacōes

$01 / 08 / 2013$

\section{Trabalho realizado na Faculdade de Medicina da Universidade Federal do Rio Grande - FURG - Rio Grande (RS), Brasil.}

IPrograma de Pós-Graduação em Ciências da Saúde da Faculdade de Medicina, Universidade Federal do Rio Grande - FURG - Rio Grande (RS), Brasil.

2Instituto Nacional de Câncer - INCA - Rio de Janeiro (RI), Brasil.

${ }^{3}$ Faculdade de Medicina, Universidade Federal do Rio Grande - FURG - Rio Grande (RS), Brasil.

Conflito de interesses: não há 


\section{Introdução}

Chlamydia trachomatis é uma bactéria gram-negativa, intracelular obrigatória ${ }^{1}$, com um ciclo de desenvolvimento bifásico que ocorre com coexistência de duas formas morfológicas distintas — os corpúsculos elementares (CE), que são extracelulares, metabolicamente inativos e infecciosos, e os corpúsculos reticulares (CR), que são intracelulares, não infecciosos e metabolicamente ativos ${ }^{2}$. A infecção por $C$. trachomatis é a mais comum doença sexualmente transmissível (DST) causada por bactéria em todo o mundo, superando a infecção gonocócica e sífilis ${ }^{3}$, constituindo um dos maiores problemas de saúde pública.

Estima-se que ocorram cerca de 92 milhões de novos casos de infecções por ano, e a maior prevalência tem sido relatada no Sudeste da Ásia (45 milhões), seguido por África Subsaariana (15 milhões) ${ }^{4}$. O Centers for Disease Control and Prevention (CDC) relatou a existência de 976.445 casos de infecções genitais causadas por C. trachomatis em 2005 nos EUA, e a prevalência em mulheres nos diversos estados variou entre 3,1 e 14,5\% . A Coordenação Nacional Brasileira de AIDS e DST relata aproximadamente 2 milhões de novos casos de infeção por $C$. trachomatis por ano ${ }^{6}$. No Brasil, não existem estudos documentando a situação real da infecção por $C$. trachomatis, estão disponíveis apenas estudos isolados em populações específicas do país ${ }^{5,7}$. A prevalência de infecção por $C$. trachomatis no Brasil varia de $0,60^{8}$ a $20,7 \%{ }^{9}$.

O maior desafio de firmar o diagnóstico dessa infecção é a verificação de que 70 a $80 \%$ das mulheres infectadas são assintomáticas ${ }^{10} \mathrm{e}$ isso pode levar a sequelas graves, como a doença inflamatória pélvica (DIP), gravidez ectópica e a infertilidade ${ }^{11}$.

Os fatores de risco para aquisição de infecção por $C$. trachomatis descritos na literatura são: gestação, atividade sexual precoce, múltiplos parceiros, relações sexuais sem uso de preservativo, indivíduos portadores de outras DSTs, fatores socioeconômicos, hábito de fumar, falta de conhecimento sobre DST, ser solteiro e uso de contraceptivos hormonais ${ }^{10}$.

Em razão da escassez de trabalhos relacionados à infecção genital causada por $C$. trachomatis no Brasil, especialmente nos Estados do sul do País, o presente estudo teve como objetivo determinar a prevalência e os fatores de risco associados à infecção por $C$. trachomatis em amostras endocervicais de mulheres de uma cidade do Estado do Rio Grande do Sul.

\section{Métodos}

Estudo transversal com amostragem por conveniência realizada no período de setembro 2008 a março de 2010. O número da amostra foi calculado utilizando o programa
Epi-info 6.04 ${ }^{\hat{a}}$. Com base na média de 300 atendimentos no período de 2 anos no ambulatório de Ginecologia e Obstetrícia Geral do Hospital Universitário da Universidade Federal do Rio Grande (HU-FURG) e nas referências encontradas na literatura, que variam de 0,6 a $20 \%$ para encontrar uma prevalência de $10 \%$, com um erro de $+/-5 \%$ e um nível de confiança de 99\%, seriam necessárias 193 amostras.

Foram incluídas as pacientes com idade igual ou superior a 14 anos que durante a consulta eram submetidas à coleta do exame citopatológico do colo uterino. Foram excluídas do estudo participantes sem amostra suficiente para realizar a extração do ácido desoxirribonucleico (DNA). No total, 200 mulheres participaram do estudo e todas responderam a um questionário autoaplicável por meio do qual foram obtidas informações sociodemográficas (cor da pele, idade, escolaridade, renda, estado civil) e ginecológicas (início da vida sexual, número de parceiros sexuais, uso de preservativo e sintomas de DST).

Amostras de secreção endocervical das participantes foram coletadas, com o auxílio de uma escova Vagispec ${ }^{\circledR}$, para posterior análise molecular. As amostras foram condicionadas em tubos criogênicos contendo $1 \mathrm{~mL}$ de tampão Tris-EDTA (TE) (10 mM Tris-HCl pH 8,5; 1 mM EDTA) e encaminhadas ao Laboratório de Biologia Molecular da Faculdade de Medicina (FAMED) da FURG até a etapa de extração do DNA.

O DNA genômico foi extraído das amostras utilizando o kit comercialmente adquirido: GFX Genomic Blood DNA Purification Kit, da GE Healthcare ${ }^{\circledR} /$ Amersham Biosciences ${ }^{\circledR}$. Para visualização dos fragmentos de DNA, foi feita uma eletroforese em gel de agarose $0,8 \%$.

A partir do DNA genômico extraído, um fragmento de 281 pares de bases (pb) da região do gene da proteína da maior membrana externa (MOMP) de C. trachomatis foi amplificado por meio de polymerase chaim reaction (PCR). Os primers utilizados para reação foram; CT05/06: 5'-GATAGCGAGCACAAAGAGAGCTAA-3'/5'-TTCAC ATCTGTTTGCAAAACACGGTCGAAAACAAAG-3 ${ }^{\prime 12}$. A amplificação foi realizada no termociclador $\mathrm{MJ}$ research ${ }^{\circledR}$, nas seguintes condições de ciclagem: desnaturação inicial de $94^{\circ} \mathrm{C}$ por $5 \mathrm{~min}$, seguido de 35 ciclos de $94^{\circ} \mathrm{C}$ por $30 \mathrm{~s}$, $40^{\circ} \mathrm{C}$ por $30 \mathrm{~s}, 70^{\circ} \mathrm{C}$ por $20 \mathrm{~s}$ e extensão final de $72^{\circ} \mathrm{C}$ por 4 min. Em cada reação, foi utilizado um controle positivo de C. trachomatis previamente conhecido e um controle negativo no qual não havia presença de qualquer DNA.

Para determinação do Human papillomavirus (HPV), foi realizada uma PCR aninhada. O primeiro round, o qual amplificou um fragmento de $450 \mathrm{pb}$ da região L1 do capsídeo viral, foi realizado com primers externos: MY09/11 (5'CGTCCMAARGGA WACTGATC 3') e MY11(5'GCMC AGGGWCATAAYAATGG $\left.3^{\prime}\right)^{13}$. O segundo round amplificou um fragmento de $150 \mathrm{pb}$ também da região L1 e utilizou os primers internos: GP5/6: 5'TTTGTTACTCTGGTAGATAC3'/ 
5'GAAAAATAAACTGTAAATCA $3^{\prime 14}$. A PCR foi realizada no termociclador $\mathrm{MJ}$ research ${ }^{\circledR}$ nas seguintes condições de ciclagem: $95^{\circ} \mathrm{C}$ por $9 \mathrm{~min}$, seguidos de 40 ciclos de $95^{\circ} \mathrm{C}$ por $1 \mathrm{~min}, 55^{\circ} \mathrm{C}$ por $1 \mathrm{~min}, 72^{\circ} \mathrm{C}$ por $1 \mathrm{~min}$ e $72^{\circ} \mathrm{C}$ por $5 \mathrm{~min}$, para extensão final.

Em seguida, foi realizada a eletroforese em gel de agarose 2\% para PCR CT 05/06, 1,0\% para PCR MY09/11 e $2 \%$ para PCR GP5/6. Os fragmentos foram visualizados por fluorescência UV por um sistema de fotodocumentação EDAS Kodak após terem sido banhados em brometo de etídio $(10 \mathrm{mg} / \mathrm{mL})$, e o tamanho foi comparado com o marcador de peso molecular, Leidwing Biotc ${ }^{\circledR}$ Ladder 50 pb Puls.

A portabilidade do vírus HIV foi confirmada por revisão de prontuário em que foi verificada a existência de dois exames de imunoensaio (ELISA) positivo e um confirmatório, que no nosso serviço é realizado pelo método de imunofluorescência indireta (IFI).

Para as análises estatísticas, foram utilizados os programas do software SPSS, prevendo a realização da análise bruta, do cálculo da razão de prevalência, dos intervalos de confiança e do valor $\mathrm{p}$, considerando que as diferenças foram estatisticamente significativas quando $\mathrm{p}<0,05$ e utilizando o teste exato de qui-quadrado. A análise multivariada foi realizada no programa SPSS com regressão de Poisson, sendo seguida de um modelo hierarquizado de análise, no qual foram integradas as variáveis com $\mathrm{p}=0,20$ na análise bruta. No primeiro nível, ingressaram as variáveis idade, renda familiar e escolaridade. No segundo, entraram as variáveis que representavam os fatores de risco para infecção por C. trachomatis, como idade da primeira relação sexual e portabilidade do HIV.

A presente pesquisa foi submetida e aprovada pelo Comitê de Ética em Pesquisa na Área da Saúde (CEPAS-FURG), protocolo n ${ }^{\circ} 23116.003711 / 2010-14$, parecer n ${ }^{\circ} 121 / 2010$.

\section{Resultados}

A prevalência de $C$. trachomatis foi de $11 \%$ (22 pacientes) do total de mulheres que participaram do estudo. Entre as 200 participantes, 55 (27,5\%) foram positivas para o HPV. A idade das participantes variou de 15 a 71 anos, e a média de idade foi de 33 anos; $63,5 \%$ eram brancas, $73,6 \%$ tiveram nove ou mais anos de estudo e 40,7\% viviam com renda familiar igual ou superior a três salários mínimos mensais.

Do total, 41,3\% haviam tido a primeira relação sexual com idade entre 16 e 18 anos, 80,4\% tiveram cinco ou menos parceiros sexuais na vida, 31,9 usavam camisinha como método contraceptivo, $13,3 \%$ estavam infectadas por alguma DST e 9,2\% estavam infectadas pelo HIV.

Entre as variáveis sociodemográficas associadas à presença de $C$. trachomatis com diferença significativa foram: pacientes com 8 ou menos anos de estudo apresentaram maior prevalência da infeção por $C$. trachomatis $(\mathrm{p}<0,001)$; pacientes que viviam com até 1 salário mínimo também apresentaram maior prevalência da infeção por $C$. trachomatis $(\mathrm{p}=0,005)$; aquelas que tiveram início vida sexual com 15 anos ou menos aparentaram maior prevalência de C. trachomatis $(\mathrm{p}=0,04)$. Houve correlação estatisticamente significativa entre a portabilidade do HIV e a prevalência de C. trachomatis $(\mathrm{p}<0,001)$; os dados podem ser encontrados na Tabela 1.

A análise multivariada mostrou que o maior risco de infeção por $C$. trachomatis está associado a pacientes com escolaridade $\leq 8$ anos de estudo (IC95\% 0,4-24,6; RP 3,1; $\mathrm{p}=0,02$ ) e aquelas infectadas pelo HIV (IC95\% 3,4-57,5; RP 14,1; $<<0,001$ ) (Tabela 2).

Tabela 1. Prevalência de Chlamydia trachomatis e fatores de risco associados à infecção em amostra endocervical

\begin{tabular}{|c|c|c|c|c|c|}
\hline Variável & n (\%) & $\begin{array}{c}\text { C. trachomatis } \\
\text { n (\%) }\end{array}$ & RP & IC95\% & Valor $p$ \\
\hline Idade & & & & & 0,05 \\
\hline 35 ou mais & $64(42,7)$ & $5(7,8)$ & 1 & & \\
\hline 25 a 34 & $42(28,0)$ & $2(4,8)$ & 0,6 & $0,1-3,0$ & \\
\hline 20 a 24 & $20(13,3)$ & $5(25)$ & 3,2 & $1,0-9,9$ & \\
\hline 19 ou menos & $24(16,0)$ & $5(20,8)$ & 2,7 & $0,8-8,4$ & \\
\hline Cor da pele & & & & & 0,3 \\
\hline Branca & $94(63,5)$ & $9(9,6)$ & 1,0 & & \\
\hline Não Branca & $54(36,5)$ & $8(14,8)$ & 1,5 & $0,6-3,8$ & \\
\hline Estado civil & & & & & 0,7 \\
\hline Com companheiro & $88(59,9)$ & $9(10,2)$ & 1,0 & & \\
\hline Sem companheiro & $59(40,1)$ & $7(11,9)$ & 1,2 & $0,5-2,9$ & \\
\hline Escolaridade & & & & & $<0,001$ \\
\hline$\geq 9$ anos & $103(73,6)$ & $6(5,9)$ & 1,0 & & \\
\hline$\leq 8$ anos & $37(26,4)$ & $10(27,0)$ & 4,6 & $1,8-11,9$ & \\
\hline \multicolumn{6}{|l|}{ Renda familiar* } \\
\hline Mais de três SM & $66(40,7)$ & $3(4,5)$ & 1 & & 0,005 \\
\hline Dois SM & $60(37,1)$ & $4(6,7)$ & 1,8 & $0,4-7,5$ & \\
\hline $\begin{array}{l}\text { Idade da primeira } \\
\text { relação sexual }\end{array}$ & & & & & 0,04 \\
\hline 19 anos ou mais & $34(22,7)$ & $1(2,9)$ & 1,0 & & \\
\hline 16 a 18anos & $62(41,3)$ & $7(10,9)$ & 3,8 & $0,3-29,9$ & \\
\hline 15 anos ou menos & $54(36,0)$ & $9(16,7)$ & 5,7 & $0,7-42,8$ & \\
\hline $\begin{array}{l}N^{0} \text { de parceiros nos } \\
\text { últimos seis meses }\end{array}$ & & & & & 0,3 \\
\hline Zero & $17(11,0)$ & $1(5,9)$ & 1,0 & & \\
\hline Um ou mais & $137(89)$ & $17(12,4)$ & 2,11 & $0,3-14,9$ & \\
\hline Usa preservativo & & & & & 0,1 \\
\hline $\begin{array}{l}\text { Sim } \\
\text { Não }\end{array}$ & $\begin{array}{l}74(48,7) \\
78(51,3)\end{array}$ & $\begin{array}{c}6(8,1) \\
12(15,4)\end{array}$ & $\begin{array}{l}1,0 \\
1,9\end{array}$ & $0,7-4,8$ & \\
\hline Sorologia para HIV & & & & & 0,001 \\
\hline HIV- & $178(90,8)$ & $14(7,7)$ & 1 & & \\
\hline HIV+ & $18(9,2)$ & $8(44,4)$ & 5,65 & $2,7-11,7$ & \\
\hline Sorologia para HPV & & & & & 0,6 \\
\hline HPV- & $145(72,5)$ & $15(10,3)$ & 1,0 & & \\
\hline $\mathrm{HPV}_{+}$ & $55(27,5)$ & $7(12,7)$ & 1,2 & $0,5-2,8$ & \\
\hline
\end{tabular}

RP: razão de prevalência; IC: intervalo de confiança; *Salário mínimo (SM) na época do estudo era $\mathrm{R} \$ 450,00$. 
Tabela 2. Análise multivariada dos fatores de risco associados à infecção por C. trachomatis em amostra endocervical

\begin{tabular}{|c|c|c|c|}
\hline Variável & RP & IC95\% & Valor $p$ \\
\hline \multicolumn{4}{|l|}{ Idade } \\
\hline 35 ou mais & 1 & & \\
\hline 25 a 34 & 0,3 & $0,04-2,2$ & 0,2 \\
\hline $\begin{array}{l}20 \text { a } 24 \\
19 \text { anos ou menos }\end{array}$ & $\begin{array}{l}1,5 \\
2,5\end{array}$ & $\begin{array}{l}0,2-11,6 \\
0,3-18,5\end{array}$ & $\begin{array}{l}0,6 \\
0,3\end{array}$ \\
\hline $\begin{array}{l}\text { Escolaridade } \\
\geq 9 \text { anos } \\
\leq 8 \text { anos }\end{array}$ & $\begin{array}{l}1 \\
6,0\end{array}$ & $1,26-29,0$ & 0,02 \\
\hline \multicolumn{4}{|l|}{ Renda familiar* } \\
\hline Até um SM & 3,1 & $0,4-24,6$ & 0,2 \\
\hline \multicolumn{4}{|c|}{ Idade da primeira relação sexual } \\
\hline 19 anos ou mais & 1 & & \\
\hline 16 a 18 anos & 3,4 & $0,3-33,4$ & 0,2 \\
\hline 15 anos ou menos & 1,6 & $0,1-18,1$ & 0,7 \\
\hline \multicolumn{4}{|l|}{ Portabilidade do HIV } \\
\hline HIV- & 1 & & \\
\hline HIV + & 14,1 & $3,4-57,5$ & $<0,001$ \\
\hline
\end{tabular}

\section{Discussão}

No presente estudo, a prevalência de $C$. trachomatis foi de $11 \%$. Estudo realizado feito no serviço de saúde pública na Bahia, utilizando o teste de amplificação do DNA na urina por meio da técnica de Ligase chain reaction (LCR) também encontrou prevalência de $11,4 \%{ }^{15,16}$. No Brasil, encontramos taxas de infecção por $C$. trachomatis que variam de $0,6 \%$ em amostra de urina de mulheres que usam serviço de saúde comunitária de Porto Alegre por meio de $\mathrm{PCR}^{8}, 6,1 \%$ em amostra cervical de mulheres que usam serviço privado de Ginecologia em Fortaleza usando captura híbrida $\mathrm{II}^{17}$ a $9,4 \%$ em amostra cervicovaginal de gestantes de seis cidades brasileiras usando captura híbrida ${ }^{7}$ Frequências mais elevadas que as observadas: $12,2 \%$ em amostra de urina de adolescente de Vitória mediante a técnica LCR ${ }^{18} ; 28,7 \%$ em amostra cervical de mulheres usando PCR em Manaus 9 No presente trabalho, pode-se observar que a prevalência de $C$. trachomatis foi semelhante à encontrada em vários outros estudos realizados no Brasil apesar das diversas técnicas para detecção em amostras biológicas diferentes.

Os principais fatores de risco para infecção por $C$. trachomatis foram: idade igual ou inferior a 24 anos, renda familiar, idade da primeira relação sexual, escolaridade e portabilidade do vírus HIV. Na literatura, os maiores casos de infecção por $C$. trachomatis são encontrados em países em desenvolvimento, afetando pessoas mais pobres $^{7,8}$. Este trabalho foi realizado em hospital público, e a maioria da população que usa esse sistema de saúde é economicamente desfavorecida. Assim, a renda familiar de até um salário mínimo e a baixa escolaridade foram fatores de risco para infecção por $C$. trachomatis. Para esclarecer o papel da renda familiar e da escolaridade sobre a prevalência de $C$. trachomatis, seria importante a realização de um estudo em população da mesma região que usa somente o sistema de saúde privado.

Início da vida sexual com 15 anos ou menos foi um preditor para infecção por $C$. trachomatis. Relato de início precoce da vida sexual e alta prevalência de $C$. trachomatis também já foram relatados na literatura ${ }^{10,19}$, porém mais estudos são necessários para explicar quais os mecanismos fisiológicos envolvidos na sinalização de infecção por $C$. trachomatis em pacientes que têm início da vida sexual precoce.

$\mathrm{Na}$ análise multivariada, as variáveis que persistiram associadas significativamente à infecção por $C$. trachomatis foram a escolaridade e a portabilidade do HIV. As pacientes com oito ou menos anos de estudo tiveram mais chance de infecção por $C$. trachomatis quando comparadas com aquelas que tiveram mais anos de estudo. Para reverter essa situação, o acesso à educação e à informação assumiria um papel fundamental na prevenção e na promoção à saúde de forma geral, fundamental em um país em crescimento como o Brasil.

Pacientes infectados por HIV apresentaram maior prevalência de $C$. trachomatis. Esse resultado está em conformidade com outros trabalhos que relatam uma correlação significativa entre a infecção pelo HIV e a prevalência de $C$. trachomatis $^{20,21}$. A epidemiologia combinada dessas infecções pode ser parcial em razão de as DSTs, incluindo C. trachomatis e HIV, terem fatores de risco sexual e comportamental semelhantes. As possíveis relações entre a infecção por $C$. trachomatis e o HIV seriam que a patogenia intracelular invasiva da $C$. trachomatis pode causar danos substanciais para a camada epitelial genital que podem facilitar a infecção por HIV. Além disso, as alterações imunológicas, decorrentes da infecção por HIV, podem favorecer infecção por $C$. trachomatis ${ }^{21}$. É importante salientar que o diagnóstico precoce de DST como a $C$. trachomatis evita o risco de transmissão de outras DST e sequelas graves. Seria de extrema importância a implementação do diagnóstico de $C$. trachomatis como parte dos testes de rotina em todos os serviços de Ginecologia e Obstetrícia.

Apesar de a infecção por HPV não ter se mostrado significativa como fator de risco para aquisição de $C$. trachomatis nesta pesquisa, é importante salientar sua alta prevalência e sua importância como agente etiológico para o desenvolvimento do câncer cervical ${ }^{22}$.

Uma limitação deste estudo é o emprego de questionário autoaplicável, no qual informações importantes podem 
ser omitidas pelas participantes pelo receio de quebra de sigilo, podendo interferir diretamente nos resultados.

Em conclusão, a prevalência de $C$. trachomatis neste estudo foi semelhante a outros estudos feitos no Brasil, e fatores sociodemográficos e a presença do HIV são fatores de risco associados à infecção por $C$. trachomatis. Diante desses dados, são necessários mais estudos para se conhecer melhor a real situação em relação à prevalência de infectados por $C$. trachomatis e, futuramente, o desenvolvimento de pesquisas visando à maior informação à população em geral dos riscos, da forma de transmissão e principalmente de medidas de prevenção dessa DST, bem como a pesquisa para o desenvolvimento de vacinas eficazes contra $C$. trachomatis.

\section{Agradecimentos}

À Universidade Federal do Rio Grande pela oportunidade do desenvolvimento e financiamento desta pesquisa, ao Laboratório de Biologia Molecular da Faculdade de Medicina e ao Conselho Nacional de Desenvolvimento Científico e Tecnológico (CNPQ) pelo apoio financeiro.

\section{Referências}

1. Swanson KA, Taylor LD, Frank SD, Sturdevant GL, Fischer ER, Carlson JH, et al. Chlamydia trachomatis polymorphic membrane protein $D$ is an oligomeric autotransporter with a higher-order structure. Infect Immun. 2009;77(1):508-16.

2. Seadi CF, Oravec R, Poser BV, Cantarelli VV, Rossetti ML. Diagnóstico laboratorial da infecção pela Chlamydia trachomatis: vantagens e desvantagens das técnicas. J Bras Patol Med Lab. 2002;38(2): 125-33.

3. Salfa MC, Latino MA, Regine V, De Maria D, De Intinis G, Camoni $\mathrm{L}$, et al. Prevalence and determinants of Chlamydia trachomatis infection among sexually active women in Turin, Italy. IJPH. $2011 ; 8(3): 295-301$.

4. Agrawal T, Vats V, Salhan S, Mittal A. The mucosal immune response to Chlamydia trachomatis infection of the reproductive tract in women. J Reprod Immunol. 2009;83(1-2):173-8.

5. Oliveira ML, Amorim MMR, Souza ASR, Albuquerque LCB, Costa AAR. Infecção por Chlamydia em pacientes com e sem lesões intraepiteliais cervicais. Rev Assoc Med Bras. 2008;54(6):506-12.

6. Pereira SM, Etlinger D, Aguiar LS, Peres SV, Longatto Filho A. Simultaneous Chlamydia trachomatis and HPV infection in pregnant women. Diagn Cytopathol. 2010;38(6):397-401.

7. Jalil EM, Pinto VM, Benzaken AS, Ribeiro D, Oliveira EC, Garcia EG, et al. [Prevalence of Chlamydia and Neisseria gonorrhoeae infections in pregnant women in six Brazilian cities]. Rev Bras Ginecol Obstet. 2008;30(12):614-9. Portuguese.

8. Ramos MC, Becker D, Germany C, Sander MA, Stein A, Fagundes RA, et al. Estudo populacional de prevalência de Chlamydia trachomatis e neisseria gonorrhoeae por PCR em urina de mulheres residentes em vila popular atendida por serviço de saúde comunitária em Porto Alegre, Brasil. DST J Bras Doenças Sex Transm. 2003;15(2):20-5

9. Santos C, Teixeira F, Vicente A, Astolfi-Filho S. Detection of Chlamydia trachomatis in endocervical smears of sexually active women in Manaus-AM, Brazil, by PCR. Braz J Infect Dis. 2003;7(2):91-5.

10. Marques CAS, Menezes MLB, Coelho IMG, Marques CRC, Celestino LC, Melo MC, et al. Infecção genital por Chlamydia trachomatis em casais atendidos em ambulatório de esterilidade conjugal. DST J Bras Doenças Sex Transm. 2007; 19(1):5-10.

1 1. Carey AJ, Beagley KW. Chlamydia trachomatis, a hidden epidemic: effects on female reproduction and options for treatment. Am J Reprod Immunol. 2010;63(6):576-86.
12. Zereu M, Zettler CG, Cambruzzi E, Zelmanowicz A. Herpes simplex virus type 2 and Chlamydia trachomatis in adenocarcinoma of the uterine cervix. Gynecol Oncol. 2007;105(1):172-5.

13. Manos MM, Ting Y, Wright DK, Lewis AJ, Broker TR, Wolinsky SM. Use of polymerase chain reaction amplification for the detection of genital human papillomaviruses. Cancer Cells. 1989;7:209-14.

14. van den Brule AJ, Snijders PJ, Gordijn RL, Bleker OP, Meijer CJ, Walboomers JM. General primer-mediated polymerase chain reaction permits the detection of sequenced and still unsequenced human papillomavirus genotypes in cervical scrapes and carcinomas. Int J Cancer. 1990;45(4):644-9.

15. Codes JS, Cohen DA, Melo NA, Santos AB, Codes JJG, Silva Júnior JC, et al. [STD screening in a public family planning clinic in Brazil]. Rev Bras Ginecol Obstet. 2002;24(2): 101-6. Portuguese.

16. Codes JS, Cohen DA, Melo NA, Teixeira GG, Leal AS, Silva TJ, et al. Detecção de doenças sexualmente transmissíveis em ambientes clínicos e não clínicos na Cidade de Salvador, Bahia, Brasil. Cad Saúde Pública. 2006;22(2):325-34.

17. Eleutério RMN, Eleutério Junior J, Giraldo PC, Muniz AMV. Cervicite por Chlamydia trachomatis em mulheres sexualmente ativas atendidas em um serviço privado de ginecologia na cidade de Fortaleza. Rev Bras Anal Clin. 2007;39(4):287-90.

18. Miranda AE, Szwarcwald CL, Peres RL, Page-Shafer K. Prevalence and risk behaviors for chlamydial infection in a populationbased study of female adolescents in Brazil. Sex Transm Dis. 2004;31 (9):542-6.

19. Paul KJ, Garcia PJ, Giesel AE, Holmes KK, Hitti JE. Generation C: prevalence of and risk factors for Chlamydia trachomatis among adolescents and young women in Lima, Peru. J Womens Health (Larchmt). 2009; 18(9):1419-24.

20. Kouri V, Cartaya J, Rodríguez ME, Muné M, Soto Y, Resik $S$, et al. Prevalence of Chlamydia trachomatis in human immunodeficiency virus-infected women in Cuba. Mem Inst Oswaldo Cruz. 2002;97(8):1073-7.

21. Joyee AG, Thyagarajan SP, Reddy EV, Venkatesan C, Ganapathy $M$. Genital chlamydial infection in STD patients: its relation to HIV infection. India J Med Microbiol. 2005;23(1):37-40.

22. Barros NKS, Alves RRF, Carneiro MAS, Rabelo-Santos SH. O papel da associação das infecções por Papilomavírus humano e a Chlamydia trachomatis no desenvolvimento do câncer cervical. Rev Eletrônica Farm. 2007;4(2): 1 14-8. 